\title{
M/M/1/N Queuing System with Retention of Reneged Customers
}

\author{
Rakesh Kumar \\ School of Mathematics, Shri Mata Vaishno Devi University, Katra \\ Sub-Post Office, University Campus-182320, Jammu \& Kashmir, India \\ rakesh_stat_kuk@yahoo.co.in, rakesh.kumar@smvdu.ac.in \\ Sumeet Kumar Sharma \\ School of Mathematics, Shri Mata Vaishno Devi University, Katra \\ Sub-Post Office, University Campus-182320, Jammu \& Kashmir, India \\ sharmasumeet786@yahoo.co.in
}

\begin{abstract}
The concept of reneging has been exploited to a great extent in recent past by the queuing modelers. Reneging leads to loss of potential customers. Keeping into mind this negative impact of reneging, a new queuing model has been developed which deals with retention of reneged customers. In this paper, we have developed an $\mathrm{M} / \mathrm{M} / 1 / \mathrm{N}$ queuing model which deals with retention of reneged customers. The steady-state solution of the model has been derived and some performance measures have been obtained. The effect of the probability of customer retention on the expected system size has been studied. A comparative analysis of some queuing models has been carried out to see the impact of customer retention on the expected system size. Finally, some queuing models have been derived as particular cases of this model.
\end{abstract}

Keywords: Customer Retention, Reneging, Steady-State Solution, ComparativeAnalysis, Finite Capacity.

\section{Introduction}

In the current scenario of population explosion and globalization of international commerce and trade, the queuing problems have gained a lot of significance in the decision making process. Queuing theory has revolutionized the industry and logistics sector apart from its immense applications in many other areas like city traffic, air traffic, bio-sciences, population studies, health sector etc. Queuing with customer impatience has special significance for the business community as it has a very negative effect on the revenue generation of a firm. A customer is said to be impatient if he tends to join the queue only when a short wait is expected and tends to remain in the line if his wait has been sufficiently small. Impatience generally takes three forms. The first is balking, deciding not to join the queue at all up on arrival; the second is reneging, the reluctance to remain in the waiting line after joining and waiting, and the third is jockeying between lines when each of a number of parallel service channels has its own queue, Gross and Harris (1985). A very nice review on queuing systems with impatient customers has been presented by Wang et al (2010). He has surveyed various queuing systems according to various dimensions like customer impatience behaviors, solution methods of queuing models with impatient customers and associated optimization aspects.

The notion of customer impatience appeared in queuing theory in the work of Haight (1957). He considered a model of balking for $\mathrm{M} / \mathrm{M} / 1$ queue in which there was a greatest queue length at which an arrival would not balk. This length was a random variable 
whose distribution was same for all customers. Haight (1959) studied a queue with reneging. Ancker and Gafarian (1963a) studied M/M/1/N queuing system with balking and reneging and derived its steady state solution. Ancker and Gafarian (1963b) obtained results for a pure balking system (no reneging) by setting the reneging parameter equal to zero. Gavish and Schweitzer (1977) considered a deterministic reneging model with the additional assumption that arrivals can be labeled by their service requirement before joining the queue and arriving customers are admitted only if their waiting time in the system does not exceed some fixed amount. Robert (1979) discussed in detail the reneging phenomenon of single channel queues. Baccelli et al (1984) considered customer impatience in which a customer gives up whenever his patience or waiting time is larger than a random threshold.

Senthil Kumar and Arumuganathan (2010) study single server batch arrival retrial queue with active breakdowns, two types of repair and second optional service (SOS). The server provides preliminary first essential service (FES) to the primary arriving customers or customers from retrial group. The customer under service decides probabilistically to remain in service or join the orbit during the breakdown. Bae and Kim (2010) considered a $\mathrm{G} / \mathrm{M} / 1$ queue in which the patience time of the customers is constant. They derived the stationary distribution of the workload of the server, or the virtual waiting time by the level crossing argument. Liau (2011) developed a queuing model for estimating business loss, although business loss is quite difficult to estimate. Balking index and reneging rate are used in the model to represent different configurations of balking behaviour and reneging behaviour respectively for different queuing systems. Using balking index and reneging rate enables decision makers to have the capability to estimate the incurred business loss for different values of balking index, reneging rate and service level. Kapodistria (2011) studied a single server Markovian queue with impatient customers and considered the situations where customers abandoned the system simultaneously. $\mathrm{He}$ considered two abandonment scenarios. In the first one, all present customers became impatient and performed synchronized abandonments, while in the second scenario; the customer in service was excluded from the abandonment procedure. He extended this analysis to the $\mathrm{M} / \mathrm{M} / \mathrm{c}$ queue under the second abandonment scenario also. Kumar (2012) investigated a correlated queuing problem with catastrophic and restorative effects with impatient customers which have special applications in agile broadband communication networks.

Keeping in mind the negative impact of customer impatience on business of any firm, the concept of retention of reneged customers has been introduced. It is envisaged that a reneged customer may be convinced to stay in the queue for his service by employing certain customer retention strategies. Thus, a reneged customer may be retained in the queue for his service with probabilityq (say) and may not be retained with probability $p=(1-q)$, that is, he may not be convinced and finally abandons the queue. In this paper, a Markovian finite capacity, single-server queuing system with retention of reneged customers has been developed. The steady-state solution of the model has been derived iteratively and different performance measures have been obtained. The effect of the probability of retention of reneged customers on the expected system size has been studied. 


\section{Queuing Model Description}

This model is based on the standard Markovian assumptions of inter-arrival and service times. The average arrival rate is $\lambda$ and the average service rate is $\mu$.The capacity of the system is taken as finite, say N. There is a single server. The queue discipline is firstcome, first- served (FCFS).Each customer upon arriving in the queue will wait a certain length of time (reneging time) for his service to begin. If it has not begun by then, he will get impatient and may leave the queue without getting service with probability $p$ and may remain in the queue for his service with probability $q(=1-p)$. The reneging times followexponential distribution with parameter $\xi$.

\section{Mathematical Formulation and Solution of the Model}

In this section, the mathematical framework of the queuing model has been presented. The differential-difference equations of the model have been derived by using the general birth-death arguments. These equations have been solved iteratively in steady-state in order to obtain the steady state solution.

Define,

$P_{n}(t)=$ the probability that there are $n$ customer in the system, that is, $n-1$ in the queue and one in service.

The differential-difference equations of the model are:

$$
\begin{gathered}
\frac{d P_{0}(t)}{d t}=-\lambda P_{0}(t)+\mu P_{1}(t) \\
\frac{d P_{n}(t)}{d t}=-[\lambda+\mu+(n-1) \xi p] P_{n}(t)+(\mu+n \xi p) P_{n+1}(t)+\lambda P_{n-1}(t) ; 1 \leq n \leq N-1 \\
\frac{d P_{N}(t)}{d t}=\lambda P_{N-1}(t)-[\mu+(N-1) \xi p] P_{N}(t] ; n=N
\end{gathered}
$$

In steady state, $\lim _{t-\infty} P_{n}(t)=P_{n}$ and therefore, $\frac{d P_{n}(t)}{d t}=0$ as $t-\infty$.

Thus, the steady-state equations corresponding to equations (1) - (3) are as follows:

$0=-\lambda P_{0}+\mu P_{1}$

$0=-[\lambda+\mu+(n-1) \xi p] P_{n}+(\mu+n \xi p) P_{n+1}+\lambda P_{n-1}$

$0=\lambda P_{N-1}-[\mu+(N-1) \xi p] P_{N} \ldots$

Solving recursively equations (4) $-(6)$, we get

$P_{n}=\prod_{k=1}^{n} \frac{\lambda}{\mu+(k-1) \xi p} P_{0} ; 1 \leq n \leq N-1 \ldots$

Also for $n:=N$ we get

$$
P_{N}=\prod_{k=1}^{N} \frac{\lambda}{\mu+(k-1) \xi p} P_{0}
$$

Using the normalization condition, $\sum_{n=0}^{N} P_{n}=1$, we get

$$
P_{0}=\frac{1}{\left(1+\sum_{n=1}^{N}\right.} \frac{1}{\left.\Pi_{k=1}^{n} \frac{\lambda}{\mu+(k-1) \xi p}\right)}
$$




\subsection{Measures of Performance:}

(1) The Expected System Size:

$$
\begin{aligned}
L_{S}=\sum_{n=0}^{N} n P_{n} & \\
L_{s} & =\sum_{n=1}^{N} n\left(\prod_{k=1}^{n} \frac{\lambda}{\mu+(k-1) \xi p}\right) P_{0}
\end{aligned}
$$

(2) The Expected Queue Length:

$$
L_{q}=\left\{\sum_{n=1}^{N} n\left(\prod_{k=1}^{n} \frac{\lambda}{\mu+(k-1) \xi p}\right) P_{0}-\frac{\lambda}{\mu}\right\}
$$

(3) The Expected Waiting Time in the System:

$$
W_{s}^{\prime}=\frac{\left[\sum_{n=1}^{N} n\left(\prod_{k=1}^{n} \frac{\lambda}{\mu+(k-1) \xi p}\right) P_{0}\right]}{\lambda}
$$

(4) The Expected Waiting Time in the queue

$$
W_{q}^{\prime}=\frac{\left[\sum_{n=1}^{N} n\left(\prod_{k=1}^{n} \frac{\lambda}{\mu+(k-1) \xi p}\right) P_{0}\right]}{\lambda}-\frac{1}{\mu}
$$

\subsection{Variation in expected system size with the variation in probability of retention}

In this sub-section, we study the variation in expected system size with respect to the probability of retention of impatient customers. When $N=4, \lambda=2, \mu=3, \xi=0.1$; and $\mathrm{q}=0.1,0.2, \ldots ., 0.99$ the variation has been shown in the following figure.

Fig.-1

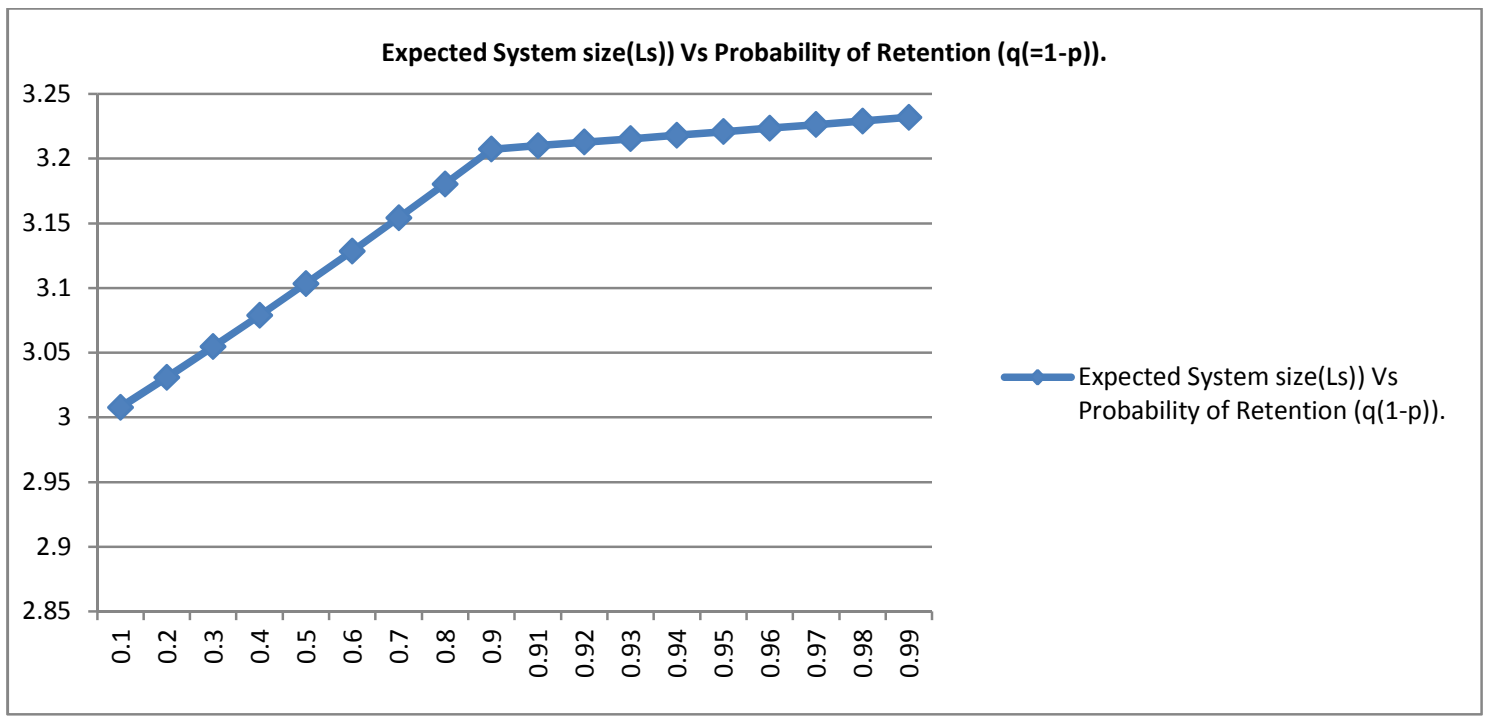


From fig. 1 we see that as we increase the probability of retention there is a steady increase in the average system size. Thus, one can study the effect of different probabilities of retention of impatient customers on the expected system size.

\section{Relative comparison of the models $M / M / 1 / N$ queue with retention, $M / M / 1 / N$ with reneging and Simple $M / M / 1 / N$ Queue}

\section{Effect of Average Arrival Rate on Expected System Size}

When $N=4, \lambda=2.0,2.1, \ldots, 2.9, \mu=3 \& \xi=0.1, q=0.6$

Fig.-2

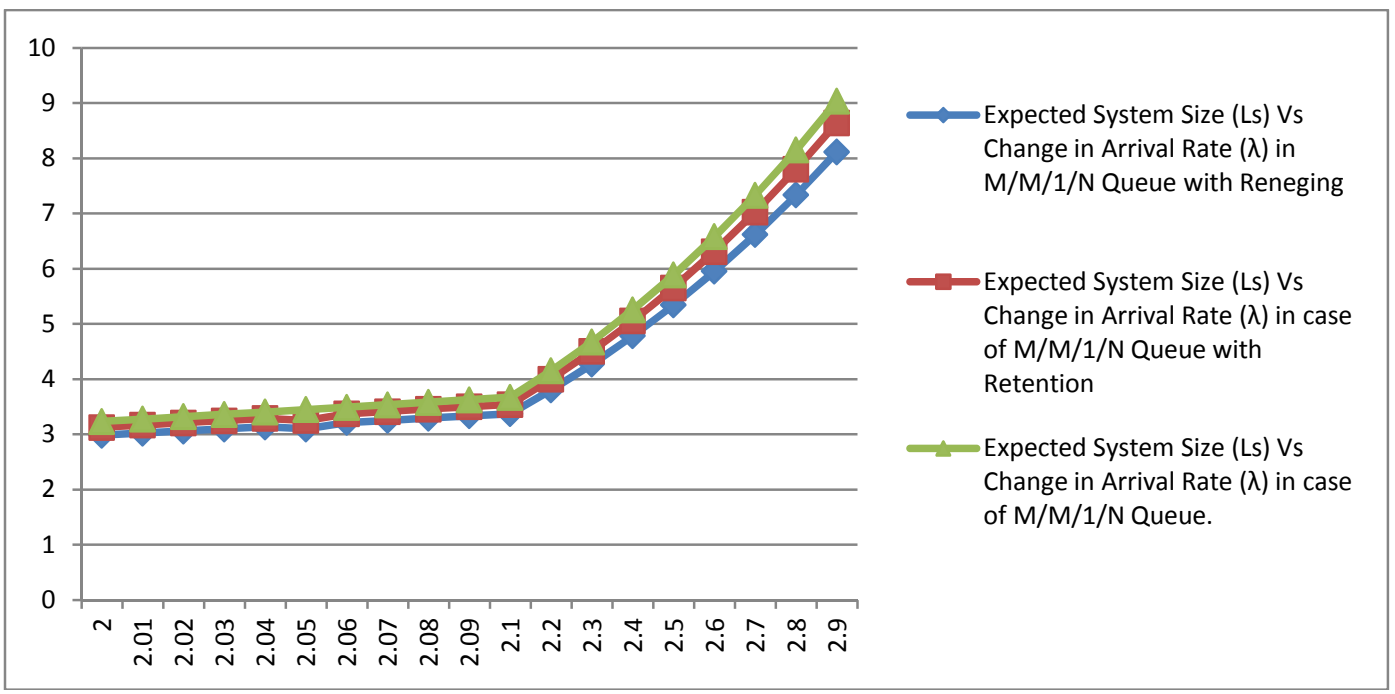

From fig.-2, it is quite evident that the average system size is higher in case of $M / M / 1 / N$ with retention than in case of $\mathrm{M} / \mathrm{M} / 1 / \mathrm{N}$ queue with reneging. Further, the average system size of $\mathrm{M} / \mathrm{M} / 1 / \mathrm{N}$ queue is higher than that of $\mathrm{M} / \mathrm{M} / 1 / \mathrm{N}$ with retention and $\mathrm{M} / \mathrm{M} / 1 / \mathrm{N}$ with reneging. Moreover, as the average arrival rate increases, the average system size also increases in all three cases.

\section{Effect of Average Service Rate on Expected System Size}

When $N=4, \lambda=2.0, \mu=2.0,2.1, \ldots . ., 3.9 \& \xi=0.1, q=0.6$

Fig.-3

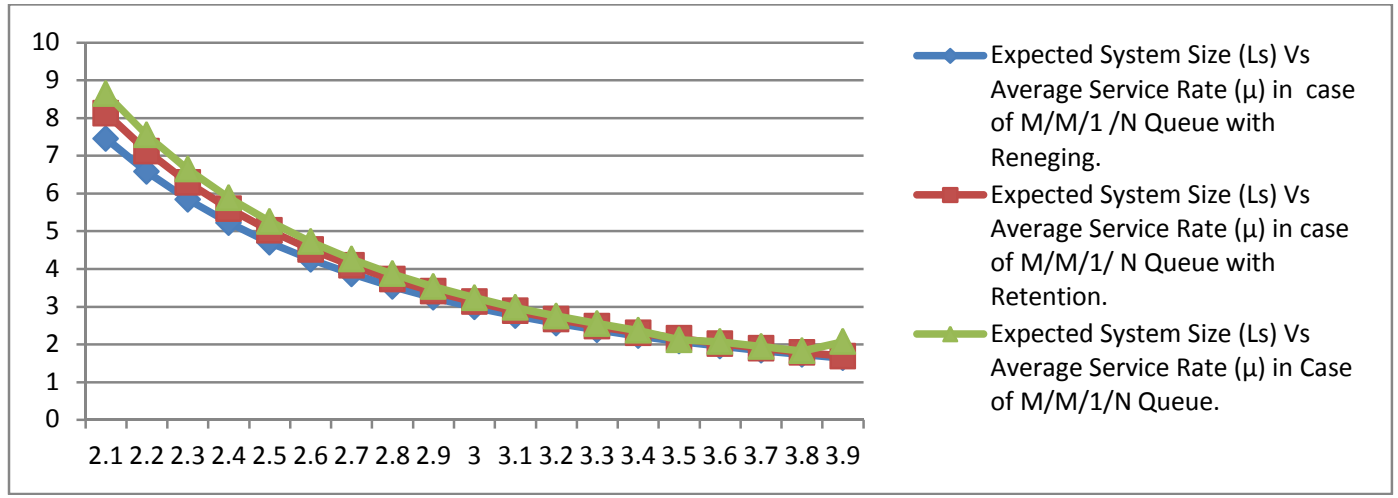


From fig-3, one can see that the average system size is higher in case of $M / M / 1 / N$ queue with retention than in case of $\mathrm{M} / \mathrm{M} / 1 / \mathrm{N}$ queue with reneging. Further, the average system size of $\mathrm{M} / \mathrm{M} / 1 / \mathrm{N}$ queue is higher than that of $\mathrm{M} / \mathrm{M} / 1 / \mathrm{N}$ with retention and $\mathrm{M} / \mathrm{M} / 1 / \mathrm{N}$ with reneging. Also, with the increase in average service rate, the average system size decreases rapidly in all the three cases.

\section{Effect of Average Reneging Rate on Expected System Size}

When $N=4, \lambda=2, \mu=3, \xi=0.01,0.02, \ldots, 0.9$ and $q=0.6$

\section{Fig.-4}

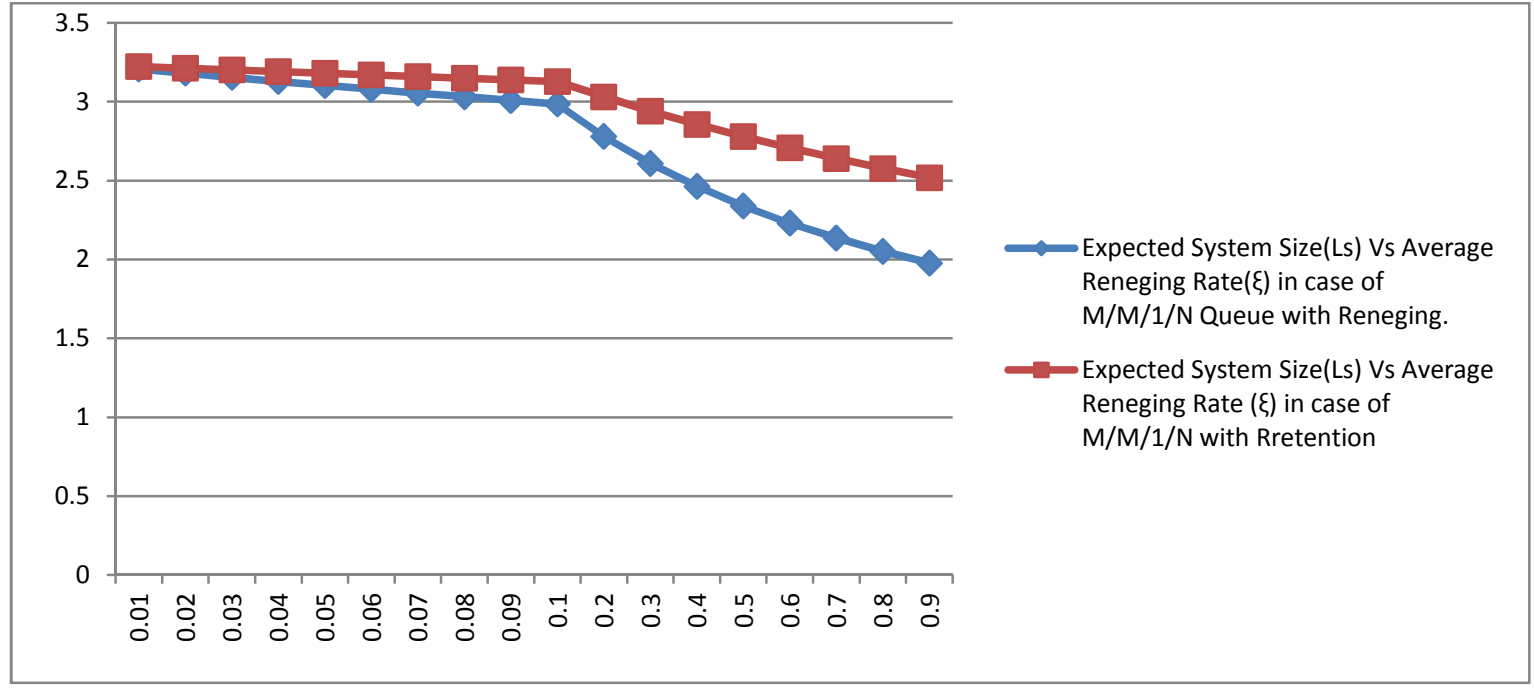

Fig. -4 shows the effect of average reneging rate on the expected system size. Expected system size decreases regularly in $\mathrm{M} / \mathrm{M} / 1 / \mathrm{N}$ with retention and $\mathrm{M} / \mathrm{M} / 1 / \mathrm{N}$ queue with reneging. Also, the average system size is higher in case of $\mathrm{M} / \mathrm{M} / 1 / \mathrm{N}$ with retention of reneged customers than in case of $\mathrm{M} / \mathrm{M} / 1 / \mathrm{N}$ queue with simple reneging.

\section{Particular Cases}

\section{(i) When the probability of retention, $q=0$}

In this case, the retention of reneged (impatient) customers is not considered. Thus, we have

$P_{n}=\prod_{k=1}^{n} \frac{\lambda}{\mu+(k-1) \xi} P_{0} \quad ; 1 \leq n \leq N-1$

Also for $n=N$ we get

$P_{N}=\prod_{k=1}^{N} \frac{\lambda}{\mu+(k-1) \xi} P_{0}$

Using the normalization condition, $\sum_{n=0}^{N} P_{n}=1$, we get

$P_{0}=\overline{\left(1+\sum_{n=1}^{N} \Pi_{k=1}^{n} \overline{\mu+(k-1) \bar{\xi}}\right)}$

That is, the model reduces to $\mathrm{M} / \mathrm{M} / 1 / \mathrm{N}$ queuing model with reneging. 


\section{(ii) When there is no reneging:}

In this case, the probability of reneging, $p=0$ which implies that $\xi=0$.

Thus, the model reduces to a simple $\mathrm{M} / \mathrm{M} / 1 / \mathrm{N}$ queue with

$P_{n}=\left(\frac{\lambda}{\mu}\right)^{n} P_{0} ; 1 \leq n \leq N$

and

$P_{0}=\frac{1-\left(\frac{\lambda}{p}\right)}{1-\left(\frac{\lambda}{p}\right)^{N+1}}$

\section{Conclusions}

In this paper, we study an $\mathrm{M} / \mathrm{M} / 1 / \mathrm{N}$ queuing model with retention of reneged customers. The steady-state solution has been obtained. Some particular cases of the model have been discussed. This model may be of great importance to the businesses facing the serious problem of customer impatience. The model analysis is limited to finite capacity. The infinite capacity case of the model can also be studied. Further, the model can be solved in transient state to get time-dependent results.

\section{Acknowledgements}

Authors are immensely thankful to the anonymous referees for their constructive comments which helped us to bring this paper in the present form.

\section{References}

1. Ancker Jr., C. J. and Gafarian, A. V. (1963a). "Some Queuing Problems with Balking and Reneging I", Operations Research, 11 (1), 88-100.

2. Ancker Jr., C. J. and Gafarian, A. V. (1963b). "Some Queuing Problems with Balking and Reneging II", Operations Research, 11(6), 928-937.

3. Baccelli, F., Boyer, I. P., and Hebuterne, G. (1984). "Single-server Queues with Impatient Customers", Advances in Applied Probability, 16, 887-905.

4. Bae, J. and Kim, S. (2010). "The Stationary Workload of the G/M/1 Queue with Impatient Customers", Queuing Systems, 64, 253-265.

5. Gross, D. and Harris, C. M. (1985). "Fundamentals of Queuing theory", $2^{\text {nd }}$ Ed., Wiley, Hoboken, NJ.

6. Gavish, B. and Schweitzer, P. J. (1977). "The Markovian Queue with Bounded Waiting Time", Management Science, 23 (12), 1349-1357.

7. Haight, F. A. (1957). "Queuing with Balking”, Biometrika, 44,360-369.

8. Haight, F. A. (1959). "Queuing with Reneging”, Metrika, 2, 186-197.

9. Kapodistria, S. (2011). "The M/M/1 Queue with Synchronized Abandonments", Queuing Systems, 68, 79-109.

10. Kumar, R. (2012). "A Catastrophic-cum-Restorative Queuing Problem with Correlated Input and Impatient Customers", International Journal of Agile Systems and Management, 5, In Press. 
11. Liau, P-Y. (2011). "The Queuing Model with Balking Index and Reneging Rate", International Journal of Services and Operations Management, 10 (1) 1-12.

12. Robert, E. (1979). "Reneging Phenomenon of Single Channel Queues", Mathematics of Operations Research, 4 (2), 162-178.

13. Senthil Kumar, M. and Arumuganathan, R. (2010). "An $M^{\mathrm{X}} / \mathrm{G} / 1$ Retrial Queue with Two-Phase Service subject to active Server Breakdowns and two types of Repair" International Journal of Operational Research, 8(3), 261 - 291.

14. Wang, K., Li, N. and Jiang, Z. (2010). "Queuing System with Impatient Customers: A Review", 2010 IEEE International Conference on Service Operations and Logistics and Informatics, 15-17 July, 2010, Shandong, 82-87. 